Research Article

\title{
Experimental Study of Rainfall Infiltration in an Analog Fracture-matrix System
}

\author{
Zhen Zhong $\mathbb{D}^{1,2}$ Huicai Gao, ${ }^{1}$ and Yunjin $\mathrm{Hu} \mathbb{D}^{1,2}$ \\ ${ }^{1}$ College of Civil Engineering, Shaoxing University, Shaoxing 312000, China \\ ${ }^{2}$ Center of Rock Mechanics and Geohazards, Shaoxing University, Shaoxing 312000, China \\ Correspondence should be addressed to Yunjin Hu; huyunjin@zju.edu.cn
}

Received 4 March 2021; Accepted 30 April 2021; Published 15 May 2021

Academic Editor: Hao Zheng

Copyright (c) 2021 Zhen Zhong et al. This is an open access article distributed under the Creative Commons Attribution License, which permits unrestricted use, distribution, and reproduction in any medium, provided the original work is properly cited.

In this study, an experimental apparatus was developed to investigate unsaturated infiltration in an analog fracture-matrix system. Fracture and adjacent matrix is simulated by sands with various particle sizes. Four rainfall infiltration experiments were performed on the analog fracture-matrix system at a constant rainfall rate of $100 \mathrm{~mm} / \mathrm{h}$. The process of rainfall infiltration is measured by a combination method of tensiometers and quick moisture apparatus. The measured results reveal that fracturematrix interactions certainly exert influences on the hydraulic behaviour of unsaturated fractured matrix, and the fluid flow mainly infiltrates along the nonuniform paths within the matrix. Moreover, it is observed that the influences are greater when using a coarser sand to mimic the fracture. Specifically, the wetting phase in the matrix moves faster than that in the fracture; the fracture, therefore, acts as a vertical capillary barrier, but there exists lateral water exchange from the matrix to the fracture. Overall, this study has demonstrated the importance of fracture/matrix interactions, which should be considered when dealing with unsaturated flow through permeable matrices.

\section{Introduction}

Unsaturated zone is widely distributed in geoformation, and the fluid flow in unsaturated zone is different from that in the saturated zone. Exploration of unsaturated fluid flow in fractured rocks is of special interest to several fields, including the disposal of nuclear waste [1-3] and subsurface contaminant remediation [4-8].

The experiment plays a fundamental role in understanding flow processes in unsaturated fractured rocks, and it usually starts with the research on fluid flow through a single fracture, which has attracted substantial attentions since Lomize firstly introduced a conceptual model of using parallel glass plates to fabric open fractures [9]. Henceforth, a bunch of experiments have been carried out with remarkable progresses achieved [5, 10-15]. Nicholl and Glass developed an optical visualization technique to explore the wetting phase flow through two-phase structures (water and gas) in an analog rough-walled fracture [16]. The experimental scenario was analogous to single-phase flow through fractures, where the gas phase was invariant under steady- state conditions. The measured relative permeability was proportional to saturation (wetting phase) by third order. Brown et al. studied fluid flow paths within a fracture plane fabricated by assembling two transparent epoxy fracture surfaces together [17]. The measured fluid velocities were found to range over several orders of magnitude, and the maximum velocity was five times larger than the average velocity, indicating that channeling flow in fractured media could cause the fast breakthrough of contaminants. Su et al. performed flow visualization experiments on a fracture replica to study the liquid distribution and behaviour of seepages [18]. It was observed that infiltrating water advanced in unsaturated fractures along highly localized, nonuniform flow paths. Sun et al. developed an experimental device to explore rainfall infiltration in an artificial fracture [19]. The obtained relationships of saturation to capillary pressure and saturation to unsaturated hydraulic conductivity were both found to be nonlinear. Hu et al. developed an experimental apparatus for the determination of the unsaturated hydraulic properties of an analogous fracture [20]. They reported that there exists hysteresis between 
drainage and imbibition processes. Qian et al. conducted laboratory experiments to investigate fluid flow through a single fracture with various surface roughnesses and apertures [15]. An empirical exponential function was found to fit well the relationship between the mean velocity and the hydraulic gradient, and the fitting parameter for the exponential function was around 0.5 when hydraulic gradients ranged from 0.003 to 0.02 .

The aforementioned experiments usually neglect fracture/matrix interactions, but both field and laboratory tests have addressed the significant influence of fracture/matrix interactions on unsaturated seepage through fractured media [21-23]. Salve et al. performed liquid-release tests in highly fractured welded tuffs at Yucca Mountain. They revealed that both fracture flows and faults-matrix interactions play critical roles in the wetting-phase movement within unsaturated fractured rocks [22]. Salve et al. carried out field tests by releasing water directly into nonwelded tuffs at Yucca Mountain [23]. The field tests suggested when the matrix and fault were dry, water injected into the fault was mostly absorbed by the adjacent matrix. Moreover, although the fault started to dry immediately after one infiltration event, the surrounding matrix would retain moisture for a few months. Roels et al. further performed moisture uptake experiments in a rough fracture fabricated by two halves of a fractured brick [24]. They observed that the wetting front in the fracture with aperture of $0.01 \mathrm{~mm}$ fell behind the wetting front in the matrix. Sakaki conducted wetting experiments of a rock matrix adjacent to a single vertical fracture to understand some of the fundamental mechanisms controlling fracture-matrix interactions [25]. Results showed that the wetting front within the matrix was approximately parallel to the fracture and propagated mainly in the horizontal direction, indicating that water absorption was predominantly one-dimensional orthogonal to the vertical fracture. Rangel-German et al. developed a two-dimensional micromodel to simulate moisture uptake from fracture to surrounding matrix [26]. The experimental results showed that the water uptake rate was depended critically on the water infiltration rate through fractures. Huang et al. designed an experimental apparatus to investigate the transport of vertical flow in unsaturated fractured sandstone [13]. It was observed that the fracture enlengthened the time for the wetting phase to break through the matrix, arising the accumulation of water in the matrix around the inlet end of the fracture, which tended to enhance the local flow in the matrix.

Even though substantial efforts have been made to uncover the hydraulic behaviours of unsaturated flow through the single fracture-matrix system [7, 27-30], there is still a lack of laboratory experiments for the determination of unsaturated hydraulic properties, including suction and saturation, under fracture/matrix interactions. Here, we followed the methods of Zhong et al. [31] to present an experimental apparatus for assessing unsaturated fluid flow through an analog fracture-matrix system. Sands with different particle-size ranges are used as an analog to simulate the fracture and matrix, respectively. The apparatus is capable of taking fracture/matrix interactions into considerations; then, rainfall infiltration tests, aiming at exploring the impact of fracture/matrix interactions on the hydraulic behaviours of the unsaturated matrix and fracture, are conducted.

\section{Materials and Methods}

It is usually difficult to install time domain reflectometry (TDR) probes in rock mass to measure unsaturated hydraulic properties, and accuracy of the measurement is sensitive to the contact between the probe and the rock. Here, an experimental model is designed to use sand with different particle sizes to mimic the fracture and the matrix, respectively. This is feasible with respect to the facts that unsaturated seepage within the fracture and sand are both under joint actions of capillary and gravity forces [32]. Table 1 summarizes physical properties of sand, which was sieved into three grain size fractions: $0.315 \sim 0.63,0.63 \sim 1.25$, and $1.25 \sim 2.50 \mathrm{~mm}$, and it was classified into medium sand (MS), coarse sand (CS), and very coarse sand (VS) according to particle size ranges.

As illustrated in Figure 1, the experimental system consists of a water supply tank, a sand tank, two stainless-steel holders, and measuring devices. Figure 2 shows the detail drawing of the water supply tank and the sand tank. The water supply tank, with a length $\times$ width $\times$ height of $0.62 \mathrm{~m} \times 0.62 \mathrm{~m} \times 0.20 \mathrm{~m}$, stands on the high stainless steel holder, and it has twenty-five needles (an inside diameter of $0.5 \mathrm{~mm}$ ) uniformly distributed at its bottom to generate rainfall for the sand tank, which is situated at the low stainless steel holder right below the water supply tank. The water supply tank has a spillway at its front wall to obtain stable overflow. The sand tank $(0.42 \mathrm{~m}$ in length, $0.42 \mathrm{~m}$ in width, and $0.6 \mathrm{~m}$ in height) is made of transparent plexiglass to make direct observation of the fluid flow processes possible. The sand tank has two layers along its height, noted as the upper and lower layers. Both layers are partitioned into 3 zones, named zone F, M1, and M2, respectively, by the glass sheet (see Figures 1 and 2). Zone M1 and zone M2 of the upper layer are packed with a finer sand of height $0.35 \mathrm{~m}$ to mimic "matrix." Consequently, a vertical gap ( $30 \mathrm{~mm}$ in thick) is created in the middle, and a coarser sand is used to fill the gap (Zone F) to form "fracture" [33]. The aperture of fracture is approximately estimated by multiplying the gap thickness by coarse sand's porosity [34]. The lower layer is used to collect the water drained from the sample. During the experiments, the capillary pressure of the fractured matrix is measured by nine tensiometers (TEN, TOP Instrument Co., Ltd.) distributed at various depths within the fracture and matrix, while the saturation is monitored by quick moisture apparatus (TZS-1K, TDR, TOP Instrument Co., Ltd.) through the observation holes drilled at the backside of the sank tank.

Four experiments, indicated as experiments A, B, C, and $D$, respectively, were carried out under an invariant rainfall rate of $100 \mathrm{~mm} / \mathrm{hr}$. The top boundary of the sample could be considered as a boundary of constant flux boundary conditions $(100 \mathrm{~mm} / \mathrm{hr})$ under unsaturated flow, while four sidewalls are subjected to zero-flow boundaries. Experimental scenarios based on different combinations of sand 
TABLE 1: Physical properties of sand with various ranges of particle size.

\begin{tabular}{lcccc}
\hline Sand classification & Particle size ranges $(\mathrm{mm})$ & Bulk density $\left(\mathrm{g} / \mathrm{cm}^{3}\right)$ & Porosity & Saturated conductivity $(\mathrm{cm} / \mathrm{s})$ \\
\hline Medium sand (MS) & $0.315 \sim 0.63$ & 1.99 & 0.25 & $2.1 e-3$ \\
Coarse sand (CS) & $0.63 \sim 1.25$ & 1.72 & 0.35 & $3.5 e-3$ \\
Very coarse sand (VS) & $1.25 \sim 2.50$ & 1.59 & 0.40 & $5.2 e-3$ \\
\hline
\end{tabular}

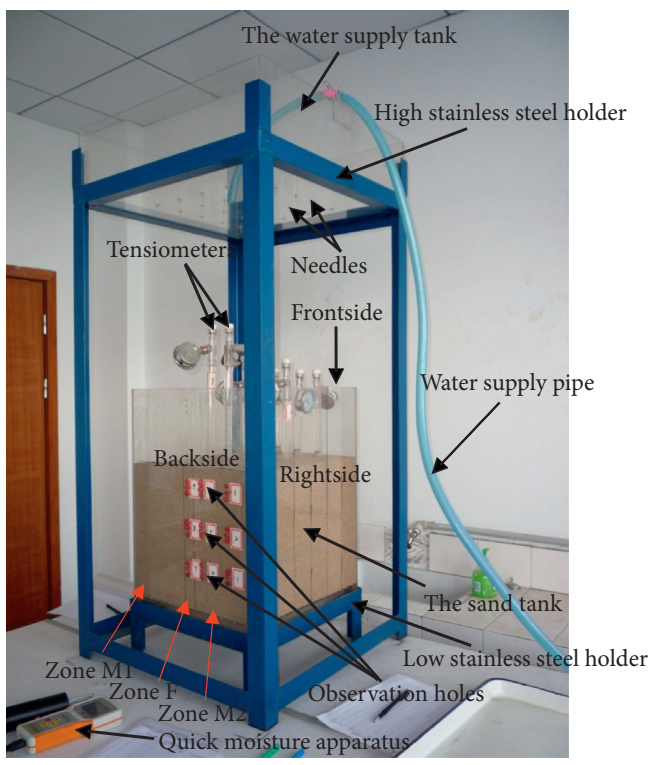

FIgURE 1: Illustration of the experimental setup.
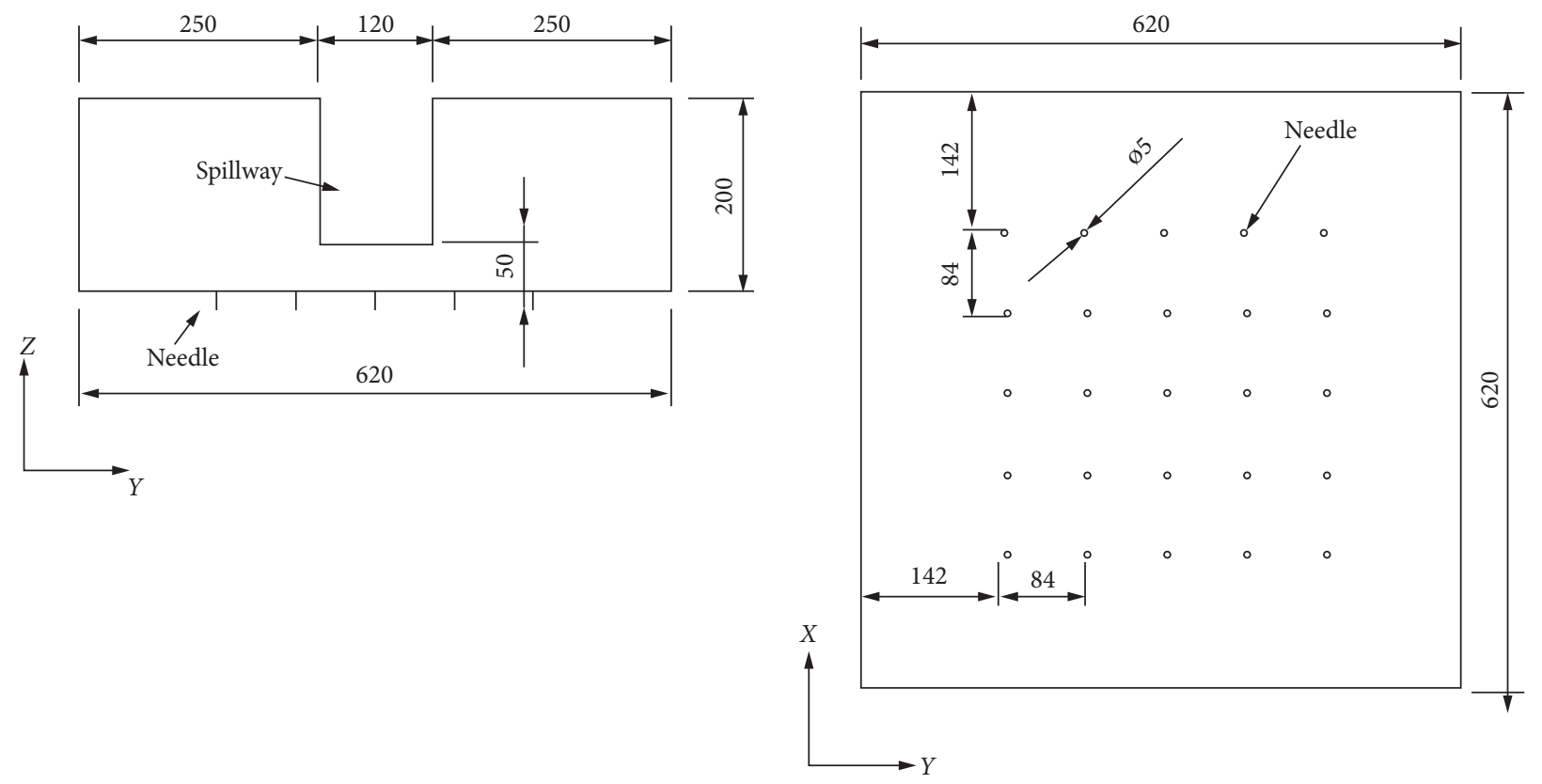

(a)

(b)

FIgURE 2: Continued. 


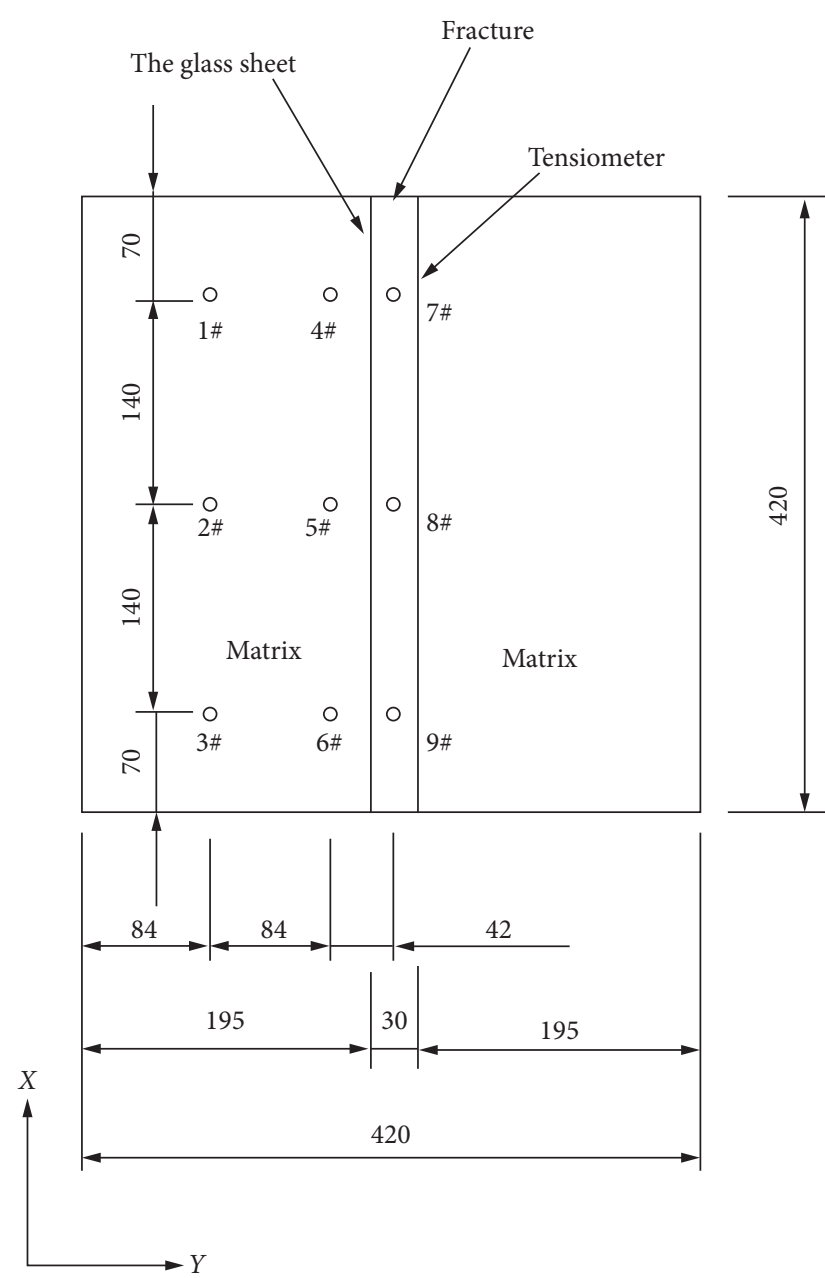

(c)

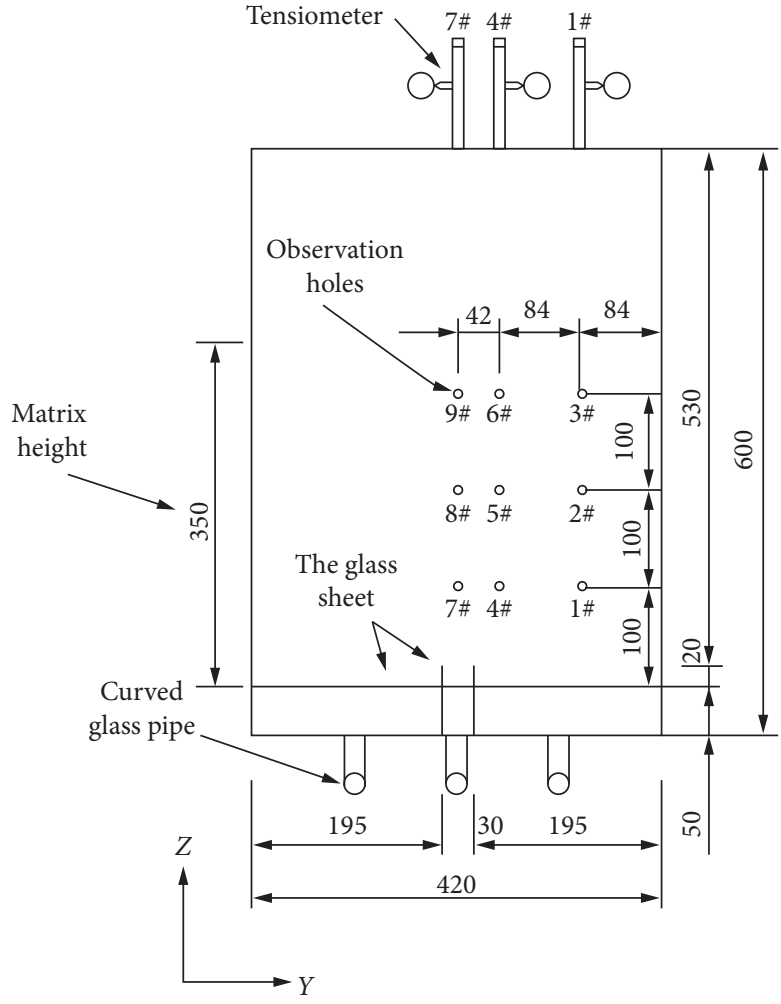

(d)

Figure 2: Annotated sketches of the experimental apparatus: (a) sectional view and (b) top view of the water supply tank; (c) top view and (d) sectional view of the sand tank, unit $(\mathrm{mm})$.

are displayed in Table 2. The initial suction and saturation are recorded using the tensiometer and quick moisture apparatus located at various depths, respectively. Water is delivered continuously to the water supply tank via a water supply pipe during the experiment, and the constant rainfall is obtained when water level keeps constant. The real-time capillary pressure and water saturation at different positions are consistently monitored during the rainfall infiltration within the fractured matrix. Effluent from the coarse- and fine-grained sand were collected separately and measured.

\section{Results and Discussion}

Experiment A, B, and C, using MS with particle sizes in the range of $0.315 \sim 0.63$ to represent the matrix, are performed to assess the influence of fracture flow on the hydraulic behaviour of the matrix. Figure 3 shows matrix suction-time curves for tensiometers located at $1 \#, 4 \#, 2 \#$, and $5 \#$, and it is obvious that fracture aperture has significant impacts on hydraulic properties of matrix, since it takes fewer time for matrix to get full saturated (suction drop to zero) as fracture aperture becomes larger. This might be caused by the joint effects of capillary barrier of fracture and fracture/matrix interactions. Specifically, it is easier for the rainfall to penetrate the matrix with smaller pores compared to the fracture with larger pores; besides, the rainfall falling on the fracture tend to accumulate around the upper boundary of the fracture induced by the capillary effect, and accumulated water is absorbed by the matrix through both sides of the fracture-matrix interface. Consequently, infiltrated rainfall mainly travel through the fractured matrix along the vertical paths within the matrix, while fracture acts as a barrier, where accumulated water will flow to the matrix. The amount of water, flowing from fracture to surrounding matrix, increases with an increase in fracture aperture, and small portion of the water, driven by the hydraulic gradient between fracture and matrix, will return to the fracture (see inset $\mathrm{A}$ in Figure 4(a)).

Matrix suction is plotted against saturation in Figure 5. The evolution of matrix suction experiences two periods as water advancing the matrix and fracture. It decreases gradually at first before drops dramatically to zero. 
TABLE 2: Experimental conditions showing different combinations of sand selected to mimic "Fracture" and "Matrix."

\begin{tabular}{lccc}
\hline Experiments & Sand used to mimic "fracture" & Fracture aperture/mm (gap thickness $\times$ sand porosity) & Sand used to mimic "matrix" \\
\hline A & MS & 7.5 & MS \\
B & CS & 10.5 & MS \\
C & VS & 12.0 & MS \\
D & CS & 10.5 & CS \\
\hline
\end{tabular}

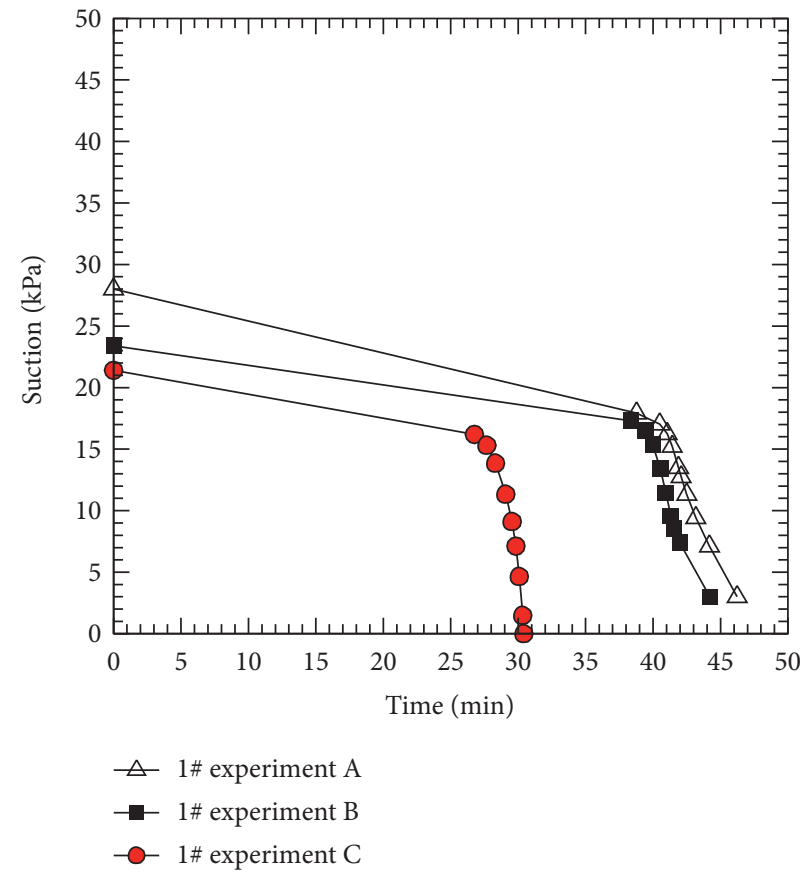

(a)

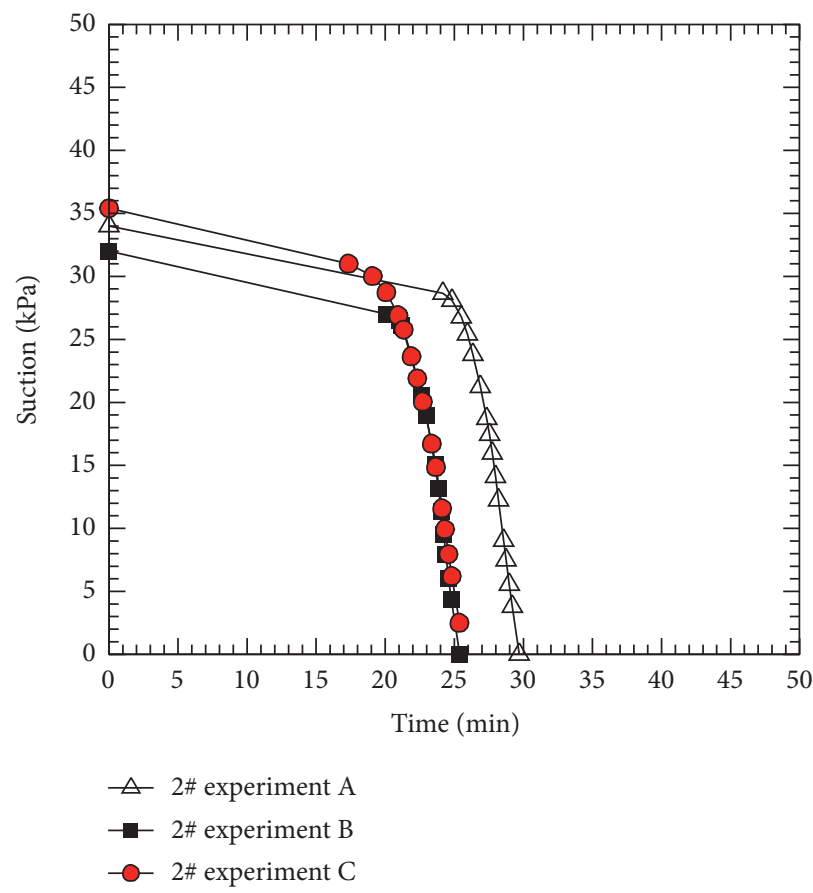

(c)

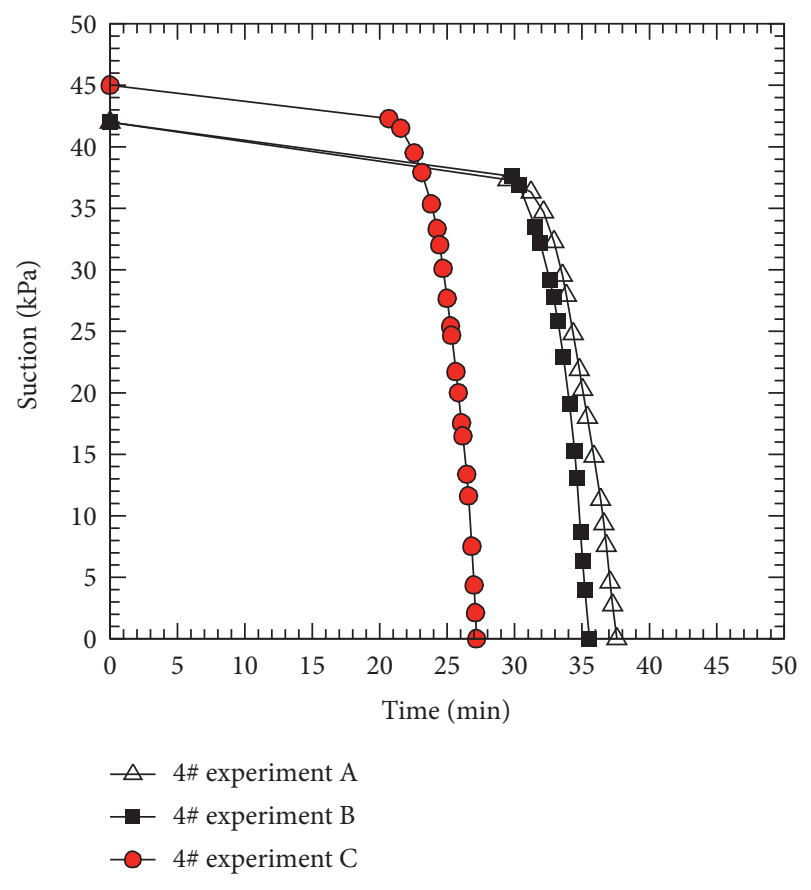

(b)

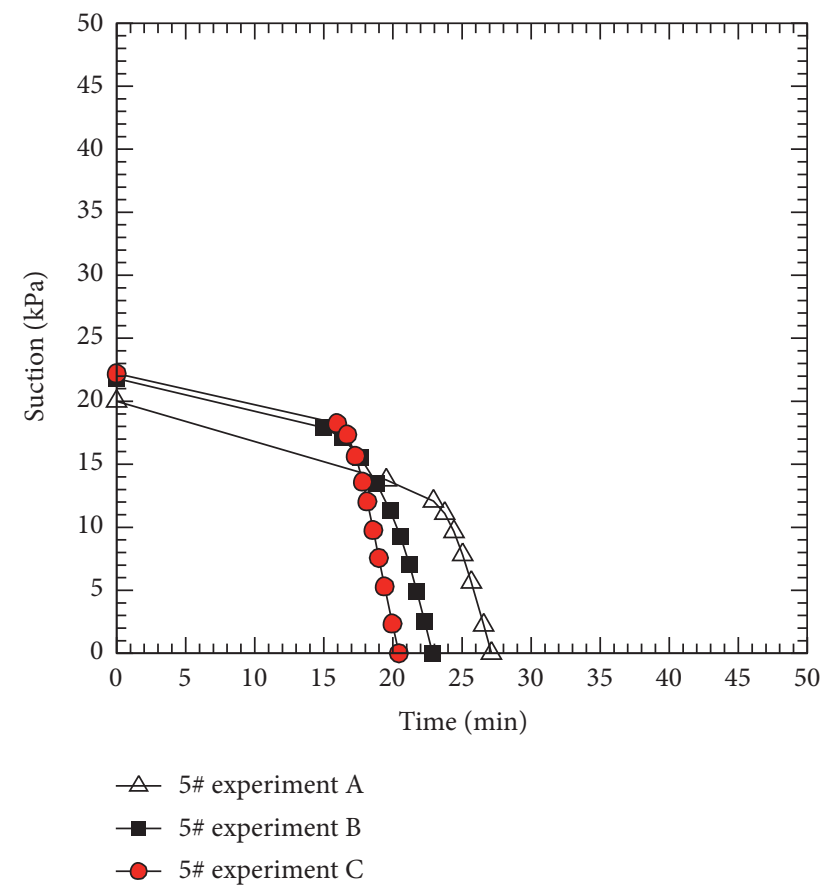

(d)

FIGURE 3: Evolution of matrix suction measured with tensiometers located at various depths of: (a) 1\# (250 mm depth); (b) 4\# (250 mm depth); (c) 2\# (150 mm depth); (d) 5\# (150 $\mathrm{mm}$ depth). 


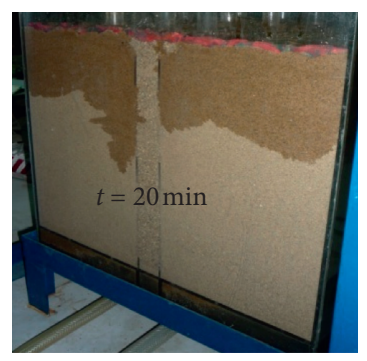

(a)

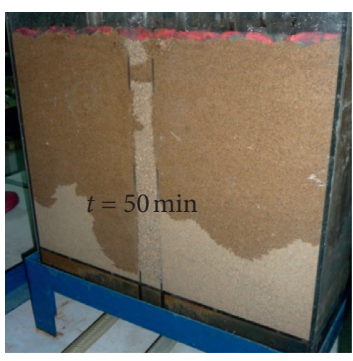

(b)

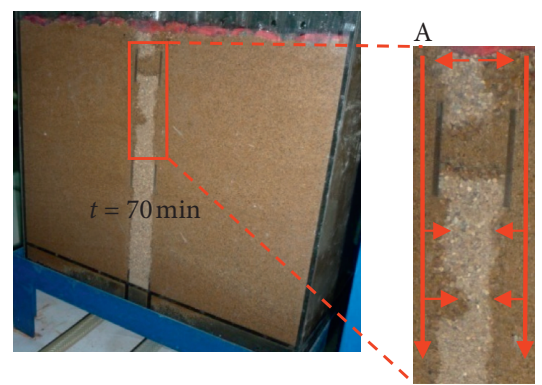

(c)

Figure 4: Vertical infiltration of water into an analog fracture-matrix system. A is the flow direction of the partial enlargement of main plot at $70 \mathrm{~min}$.

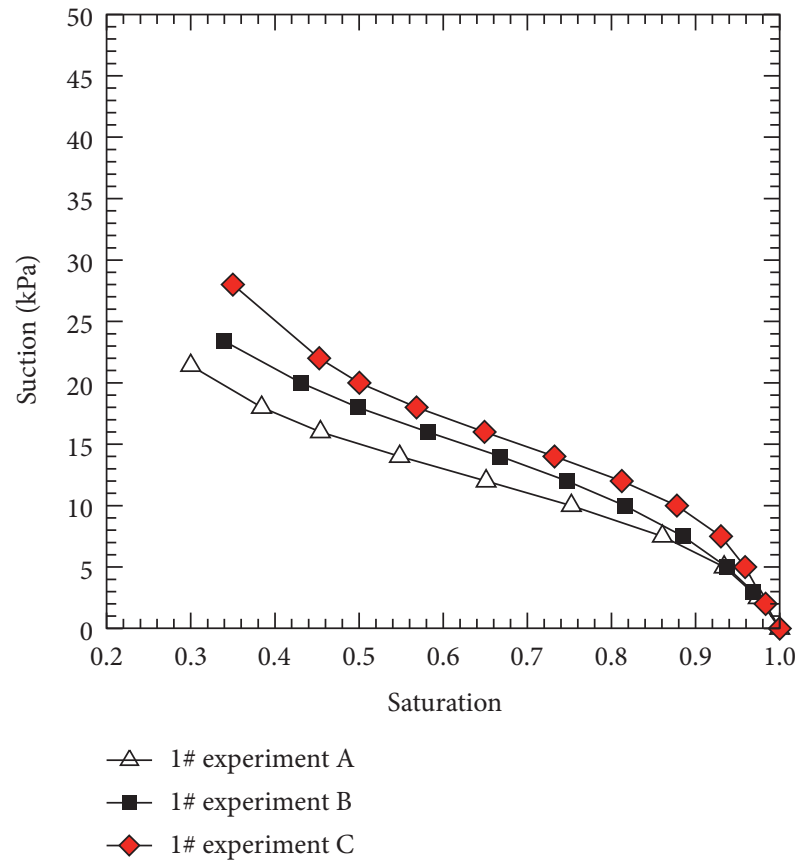

(a)

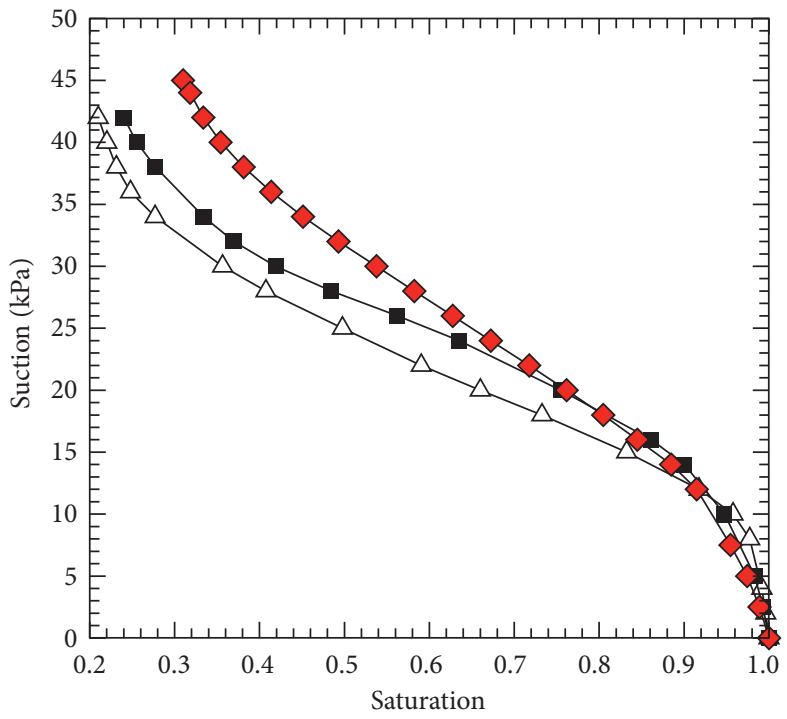

$$
\begin{aligned}
& \triangle 4 \text { A experiment } A \\
& -4 \text { \# experiment } B \\
& \neg \quad 4 \# \text { experiment } C
\end{aligned}
$$

(b)

FIgURE 5: Continued. 


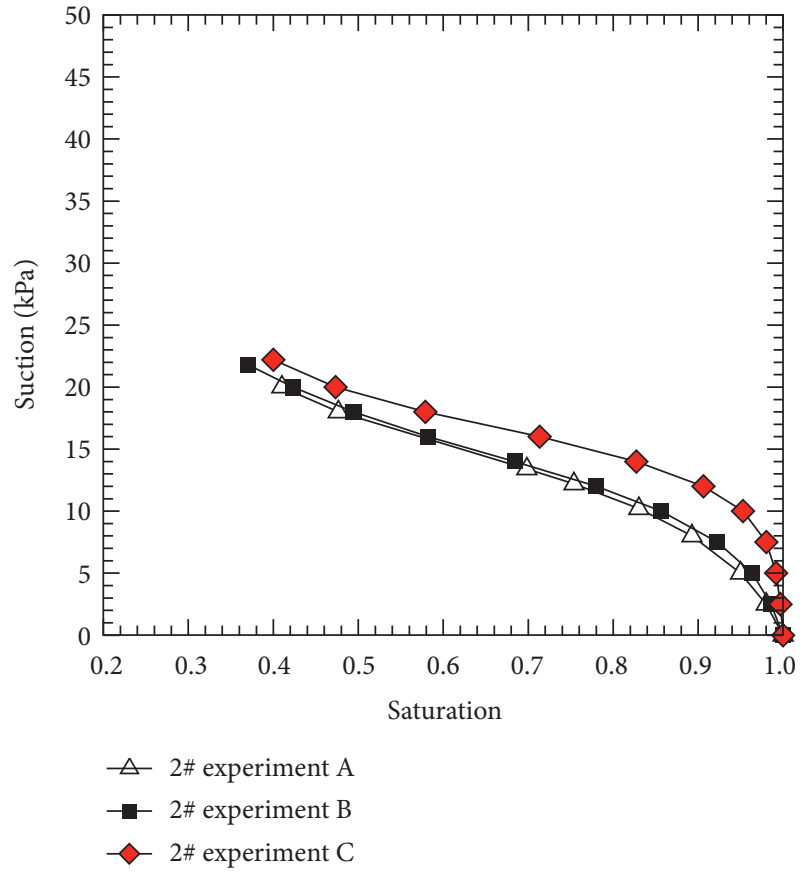

(c)

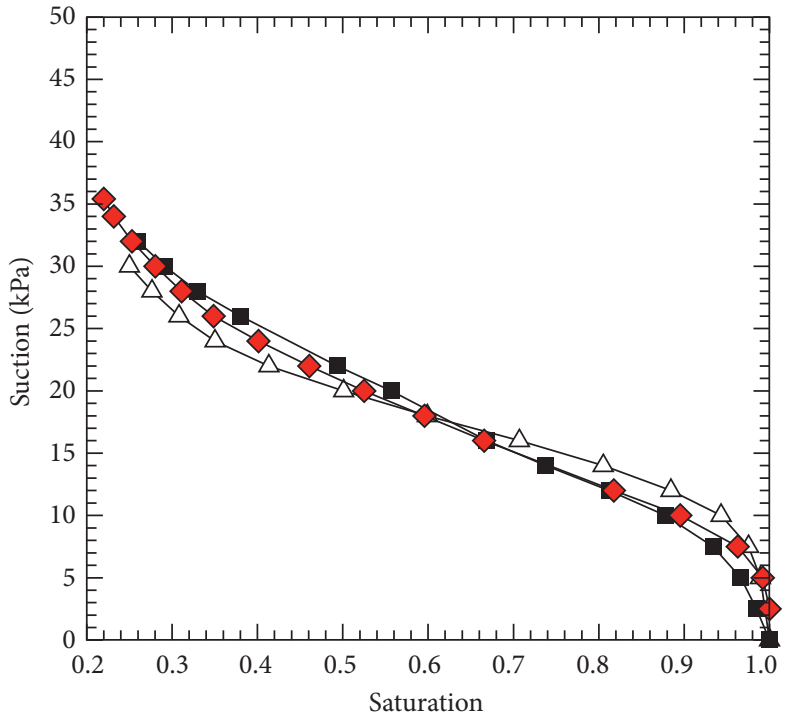

$\triangle 5 \#$ experiment $\mathrm{A}$
$\rightarrow 5 \#$ experiment $\mathrm{B}$
$\checkmark 5 \#$ experiment $\mathrm{C}$

FIGURE 5: Relationships between matrix suction and saturation tensiometers located at different depths of (a) $1 \#$ (250 mm depth), (b) $4 \#$ (250 $\mathrm{mm}$ depth), (c) 2\# (150 $\mathrm{mm}$ depth), and (d) 5\# (150 mm depth).
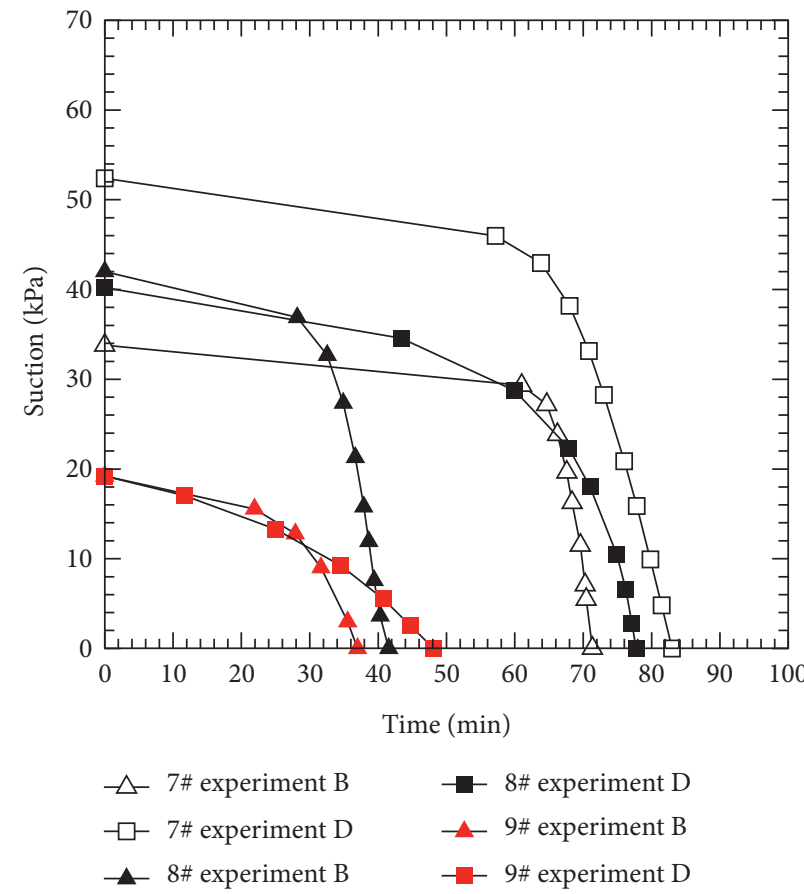

(a)

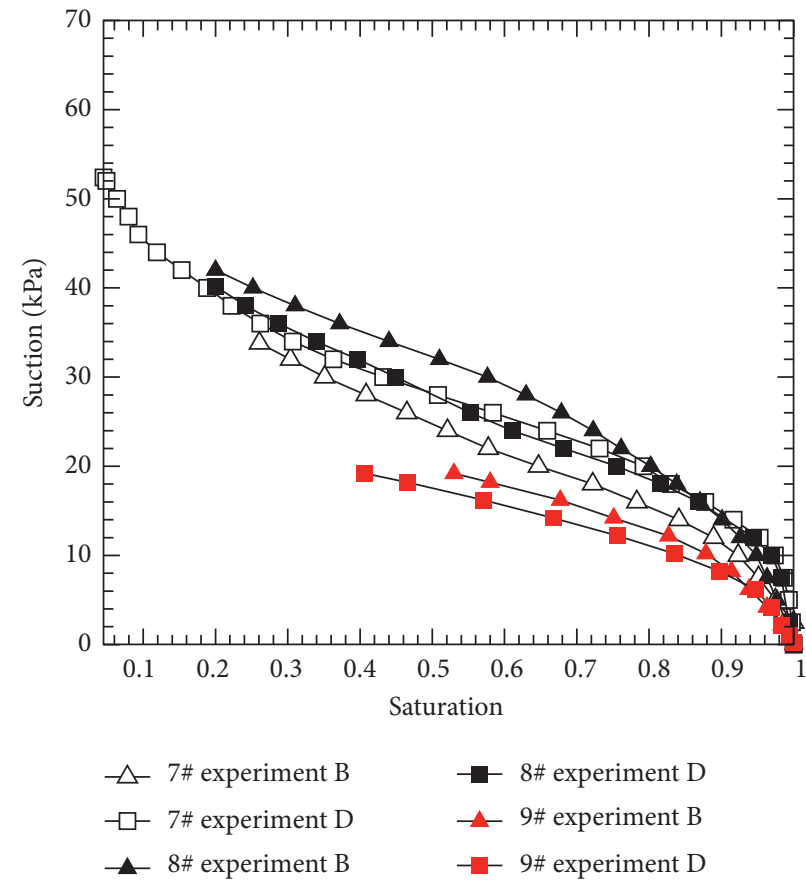

(b)

Figure 6: Plot of the experimental results for tensiometers in the fracture: (a) suction versus time; (b) suction versus saturation.

The results of experiment B and D with CS to simulate fracture are compared in Figure 6. Figure 6(a) describes evolution of capillary pressure (suction) in fracture. At the beginning of rainfall, both fracture and matrix are dry with low saturation, so infiltrated rainfall tend to occupy the matrix with smaller pores, leaving fracture with larger 
pores remain in dry. This can result in a hydraulic connection between fracture and adjacent matrix, fracture start to absorb water from matrix because of hydraulic gradient between them. The matrix provides an extra source of water for the fracture under this circumstance, and it would be easier for water to move from a finer matrix to the fracture. The finer the matrix, the quicker the suction in fracture drops to zero. Since both the fracture and matrix are actually porous media (sand), the fracture's suction-saturation curves, as indicated in Figure 6(b), are similar to those of the matrix.

The wetting front evolution of the experiment $\mathrm{C}$ is displayed in Figure 4. It is observed that infiltrated water mainly travels through unsaturated fracture-matrix system along nonuniform, localized preferential flow paths. Inset A in Figure 4 is a close-up view of rainfall infiltration at $70 \mathrm{~min}$, and it can also be concluded that flow primarily migrates downwards via the matrix in the direction parallel to the fracture, and the wetting phase within the matrix moves quicker than that in the fracture, even though some portion of water is driven back to fracture because of the hydraulic gradient between fracture and matrix. Those observations are similar with that observed in [24].

\section{Conclusions}

This paper presents a new experimental apparatus to study unsaturated flow in fractured rock. Sands with various ranges of particle sizes are chosen as analogous materials to mimic fracture and matrix, respectively. Then, rainfall experiments were performed on an analog fracture-matrix system to evaluate unsaturated infiltration processes in both "fracture" and adjacent "matrix." It is observed that unsaturated flow primarily migrate downwards via the matrix along nonuniform, localized preferential flow paths, which is quite different from the results from saturated flow, where the fracture is usually considered as a major conduit. Moreover, the preferential flow in the matrix will be enhanced since the fracture acts as a capillary barrier, and this enhancement is greater by using a coarser sand to simulate the fracture. Besides, there also exists hydraulic connection between the fracture and adjacent matrix, and fluid flow tends to transport from the fracture to the matrix due to hydraulic gradient between them, bringing in additional water supply for the fracture along the direction transversal to it. In general, it is suggested that hydraulic connection between fracture and surrounding matrix should not be ignored in unsaturated flow, especially when the matrix is permeable.

\section{Data Availability}

The data that support the findings of this study are available from the corresponding author upon reasonable request.

\section{Conflicts of Interest}

The authors declare that they have no conflicts of interest.

\section{Acknowledgments}

The authors thank Junjun Zhang from Zhejiang University for the technical assistance in the designing of the experimental system. This research was financially supported by National Natural Science Foundation of China (Grant nos. 51509154, 41977256, and 41907167), Natural Science Foundation of Zhejiang Province (Grant no. LGJ20E090001), and International Scientific and Technological Cooperation Projects of Shaoxing University (Grant no. 2019LGGH1008).

\section{References}

[1] G. P. Lu, H. H. Liu, and R. Salve, "Long term infiltration and tracer transport in fractured rocks: field observations and model analyses," Journal of Hydrology, vol. 396, no. 1-2, pp. 33-48, 2011.

[2] H.-H. Liu, "Non-Darcian flow in low-permeability media: key issues related to geological disposal of high-level nuclear waste in shale formations," Hydrogeology Journal, vol. 22, no. 7, pp. 1525-1534, 2014.

[3] I. Faoro, D. Elsworth, and T. Candela, "Evolution of the transport properties of fractures subject to thermally and mechanically activated mineral alteration and redistribution," Geofluids, vol. 16, no. 3, pp. 396-407, 2016.

[4] N. Natarajan and G. S. Kumar, "Solute transport in a coupled fracture-matrix system with sinusoidal fracture geometry," International Journal of Engineering Science and Technology, vol. 2, no. 6, pp. 1886-1992, 2010.

[5] C.-L. Cheng, E. Perfect, B. Donnelly et al., "Rapid imbibition of water in fractures within unsaturated sedimentary rock," Advances in Water Resources, vol. 77, pp. 82-89, 2015.

[6] B. R. Jones, J. L. Van Rooy, and M. A. Dippenaar, "On the differing role of contact obstacles on variably saturated flow in vertical and horizontal fractures," in IAEG/AEG Annual Meeting Proceedings, A. Shakoor and K. Cato, Eds., Springer, San Francisco, CA, USA, 2018.

[7] Z. Yang, S. Xue, X. Zheng, and Y. F. Chen, "Partitioning dynamics of gravity-driven unsaturated flow through simple T-shaped fracture intersections," Water Resources Research, vol. 55, no. 8, pp. 7130-7142, 2019.

[8] J. Qian, Z. Chen, H. Zhan, and H. Guan, "Experimental study of the effect of roughness and Reynolds number on fluid flow in rough-walled single fractures: a check of local cubic law," Hydrological Processes, vol. 25, no. 4, pp. 614-622, 2011.

[9] G. M. Lomize, Flow in Fractured Rocks, Gosemergoizdat, Moscow, Russia, 1951.

[10] R. J. Glass, M. J. Nicholl, S. E. Pringle, and T. R. Wood, "Unsaturated flow through a fracture-matrix network: dynamic preferential pathways in mesoscale laboratory experiments," Water Resources Research, vol. 38, no. 12, pp. 17-21, 2002.

[11] T. R. Wood, R. J. Glass, T. R. Mcjunkin et al., "Unsaturated flow through a small fracture-matrix network: Part 1. experimental observations," Vadose Zone Journal, vol. 3, no. 1, pp. 90-100, 2004.

[12] J. Z. Qian, Z. Chen, H. B. Zhan, and S. H. Luo, "Solute transport in a filled single fracture under non-Darcian flow," International Journal of Rock Mechanics and Mining Sciences, vol. 48, no. 1, pp. 132-140, 2011. 
[13] Z. Yong, Z. F. Zhou, Z. B. Yu, and M. J. Nicholl, "Effect of a vertical fracture on horizontal unsaturated seepage flow," Journal of Hydrodynamics, vol. 23, no. 1, pp. 127-134, 2011.

[14] T. Noffz, M. Dentz, and J. Kordilla, "Analogue fracture experiments and analytical modeling of unsaturated percolation dynamics in fracture cascades," Vadose Zone Journal, vol. 18, no. 1, pp. 1-9, 2019.

[15] J. Qian, H. Zhan, W. Zhao, and F. Sun, "Experimental study of turbulent unconfined groundwater flow in a single fracture," Journal of Hydrology, vol. 311, no. 1-4, pp. 134-142, 2005.

[16] M. J. Nicholl and R. J. Glass, "Wetting phase permeability in a partially saturated horizontal fracture," in Proceedings of the 5th International Conference on High Level Radioactive Waste Management, vol. 2, pp. 2007-2019, Las Vegas, NV, USA, May 1994.

[17] S. Brown, A. Caprihan, and R. Hardy, "Experimental observation of fluid flow channels in a single fracture," Journal of Geophysical Research: Solid Earth, vol. 103, no. B3, pp. 5125-5132, 1998.

[18] G. W. Su, J. T. Geller, K. Pruess, and F. Wen, "Experimental studies of water seepage and intermittent flow in unsaturated, rough-walled fractures," Water Resources Research, vol. 35, no. 4, pp. 1019-1037, 1999.

[19] Y. Sun, E. Z. Wang, and X. H. Chen, "Experiment studies for unsaturated flow in a single fracture under rainfall," Journal of Tsinghua University (Science and Technology), vol. 39, no. 11, pp. 14-17, 1999, in Chinese.

[20] Y. J. Hu, B. Y. Su, and G. H. Mao, "An experimental approach for determining unsaturated hydraulic properties of rock fractures," Nordic Hydrology, vol. 35, no. 3, pp. 251-260, 2004.

[21] E. R. Rangel-German and A. R. Kovscek, Matrix-fracture Interaction in Single Matrix Blocks, Stanford University Petroleum Research Institute Twenty-Third Annual Report, Stanford, CA, USA, 2000.

[22] R. Salve, J. S. Y. Wang, and C. Doughty, "Liquid-release tests in unsaturated fractured welded tuffs: I. Field investigations," Journal of Hydrology, vol. 256, no. 1-2, pp. 60-79, 2002.

[23] R. Salve, C. M. Oldenburg, and J. S. Y. Wang, "Fault-matrix interactions in nonwelded tuff of the paintbrush group at Yucca mountain," Journal of Contaminant Hydrology, vol. 6263, pp. 269-286, 2003.

[24] S. Roels, K. Vandersteen, and J. Carmeliet, "Measuring and simulating moisture uptake in a fractured porous medium," Advances in Water Resources, vol. 26, no. 3, pp. 237-246, 2003.

[25] T. Sakaki, The role of fracture-matrix interaction in drying of unsaturated fractured rock experiments and modeling, Ph.D. thesis, University of Colorado, Denver, CO, USA, 2004.

[26] E. R. Rangel-German and A. R. Kovscek, "A micromodel investigation of two-phase matrix-fracture transfer mechanisms," Water Resources Research, vol. 42, no. 3, p. W03401, 2006.

[27] F. Ren, G. Ma, Y. Wang, T. Li, and H. Zhu, "Unified pipe network method for simulation of water flow in fractured porous rock," Journal of Hydrology, vol. 547, pp. 80-96, 2017.

[28] J. R. Nimmo, K. M. Creasey, K. S. Perkins, and B. B. Mirus, "Preferential flow, diffuse flow, and perching in an interbedded fractured-rock unsaturated zone," Hydrogeology Journal, vol. 25, no. 2, pp. 421-444, 2017.

[29] B. Xiao, X. Zhang, W. Wang et al., "A fractal model for water flow through unsaturated porous rocks," Fractals, vol. 26, no. 2, Article ID 1840015, 2018.

[30] X. Li and D. Li, "A numerical procedure for unsaturated seepage analysis in rock mass containing fracture networks and drainage holes," Journal of Hydrology, vol. 574, pp. 23-34, 2019.

[31] Z. Zhong, Y. J. Hu, X. H. Zhang, and G. L. Chen, "Laboratory study of unsaturated flow in a single fracture-matrix system: a conceptual model," Applied Mechanics and Materials, vol. 212-213, pp. 377-382, 2012.

[32] J. E. Houseworth, "An analytical model for solute transport in unsaturated flow through a single fracture and porous rock matrix," Water Resources Research, vol. 42, no. 1, Article ID W01416, 2006.

[33] R. C. Starr, R. W. Gillham, and E. A. Sudicky, "Experimental investigation of solute transport in stratified porous media, 1 . The nonreactive case," Water Resources Research, vol. 21, no. 7, pp. 1035-1041, 1985.

[34] Y.-S. Wu, M. Ye, and E. A. Sudicky, "Fracture-flow-enhanced matrix diffusion in solute transport through fractured porous media," Transport in Porous Media, vol. 81, no. 1, pp. 21-34, 2010. 